\title{
Caracterização do mercado consumidor de sucos prontos para o consumo
}

\author{
Characterization of the ready to drink juice consumer market
}

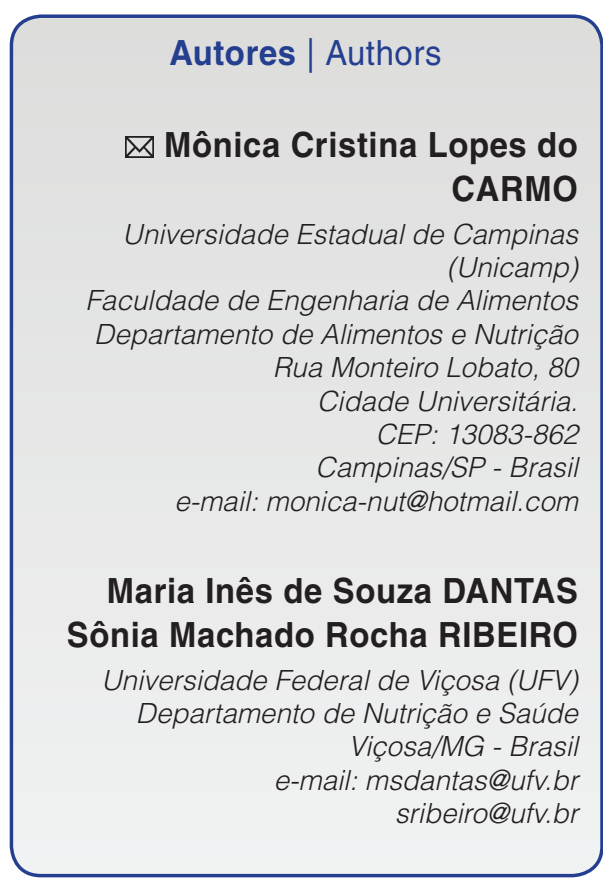

Autor Correspondente / Corresponding Author

Recebido / Received: Jun. 02, 2014 Aprovado / Approved: Fev. 27, 2015

\section{Resumo}

O mercado brasileiro de sucos prontos para beber está em franca expansão, acompanhando a tendência mundial de consumo de bebidas saudáveis e saborosas. O presente trabalho objetivou traçar o perfil dos consumidores de sucos industrializados da cidade de Viçosa, MG. A pesquisa foi exploratória, descritiva direta e estruturada por meio de uma amostragem aleatória simples com a aplicação de questionários estruturados com abordagem quantitativa, composta por 292 consumidores entrevistados; deles: 32\% consomem algum tipo de suco de três a cinco vezes por semana; $72 \%$ preferem o suco tradicional ao light/diet; a primeira opção de sabor mais citada foi o de laranja. Sabor, qualidade e validade foram critérios considerados extremamente importantes na escolha de sucos industrializados pela maioria dos entrevistados. Cerca de $45 \%$ incluíram o preço nessa categoria, enquanto a embalagem e a praticidade foram consideradas muito importantes por $39 \%$ e $44 \%$, respectivamente. Apenas $33 \%$ dos participantes consideraram o valor nutricional e a marca como muito importantes na aquisição de sucos. Metade dos consumidores afirma ler as informações nutricionais contidas nos rótulos. As marcas preferidas foram a Del Valle (22\%; $n=63)$ e Tial (21\%; $n=59)$. Sabor, qualidade e confiança foram os principais quesitos apontados para a preferência pela marca. Percebe-se que um fator comum aos consumidores de sucos prontos refere-se à saudabilidade, fator considerado fonte de satisfação unânime entre os pesquisados na etapa qualitativa. O mercado de sucos apresenta um enorme potencial a ser explorado.

Palavras-chave: Sucos Industrializados; Marketing; Mercado consumidor; Bebidas.

\section{Summary}

The Brazilian market for ready to drink juices is expanding with the worldwide trend for the consumption of healthy and tasty drinks. The present study aimed to determine the profile of consumers of processed juices in Viçosa/MG/Brazil. The research was exploratory, descriptive, direct and structured by way of simple random sampling with structured questionnaires, showing a quantitative approach. A total of 292 consumers were surveyed of which $32 \%$ drank some kind of juice 3 to 5 times per week, $72 \%$ preferred traditional to light/diet juices, and the first option most cited for flavour was orange. The taste, quality and shelf-life were considered extremely important by most respondents in choosing industrialized juices. About $45 \%$ included the price in this category, while packaging and practicality were considered very important by $39 \%$ and $44 \%$, respectively. Only $33 \%$ of the participants considered the nutritional value and brand as very important in the acquisition of juices. Half of the consumers reported reading the nutritional information on the labels. The preferred brands were Del Valle (22\%, $n=63)$ and Tial $(21 \%, n=59)$. The taste, quality and reliability were the key questions involved in brand preference. One common factor perceived amongst the consumers of ready to drink juices referred to healthiness, a factor considered a unanimous source of satisfaction amongst the respondents in the qualitative phase. The juice market has a huge potential to be explored.

Key words: Industrialized juices; Marketing; Consumer market; Beverages. 


\section{Introdução}

A cada dia mais a preocupação com a saúde se evidencia no comportamento da população. A busca pela qualidade de vida se estende aos cuidados com a alimentação, caracterizado por uma crescente demanda por produtos saudáveis e com características nutricionais e sensoriais próximas dos alimentos in natura. Dessa forma, a indústria alimentícia está mais atenta aos desejos do consumidor e investe na formulação de novos produtos, concentrando o marketing no apelo à vida saudável (WANSINK, 2004; MACHADO, 2013).

O mercado brasileiro de sucos prontos para beber está em franca expansão, acompanhando a tendência mundial de consumo de bebidas saudáveis, convenientes e saborosas. Sucos de fruta prontos para beber são considerados bebidas refrescantes, capazes de saciar a sede, ao mesmo tempo que respondem ao apelo por produtos naturais e agregam vantagens nutricionais, o que contribui para sua grande aceitação (FERRAREZI, 2008; FERREIRA e ALCÂNTARA, 2013).

É neste contexto que se verifica o aumento do consumo de bebidas não alcoólicas e não carbonatadas, tais como sucos (prontos, concentrados, entre outros), chás e águas. O segmento de sucos prontos para o consumo no mercado brasileiro apresenta constante crescimento. Os sucos prontos estão entre as bebidas que mais crescem no setor das não alcoólicas. Segundo os fabricantes, essa alta demanda é explicada pela crescente procura por produtos naturais e de rápido preparo (SEBRAE, 2006).

O estudo do comportamento do consumidor é fundamental para entender o que o leva a consumir ou não um determinado produto e quais fatores estão envolvidos no processo de compra de um alimento. Assim, a pesquisa de mercado representa uma ferramenta bastante útil para elucidar o comportamento dos consumidores de alimentos (DAGEVOS, 2005; SOLOMON, 2008; ENDO et al., 2009; DIAS et al., 2013).

O presente trabalho teve por objetivo traçar o perfil dos consumidores de sucos industrializados da cidade de Viçosa, MG.

\section{Material e métodos}

A pesquisa foi realizada após consentimento dos órgãos competentes e aprovação pelo Comitê de Ética na Pesquisa com Seres Humanos da Universidade Federal de Viçosa, sob o número 014/2006. Os entrevistados foram informados do estudo e assinaram o Termo de Consentimento Livre e Esclarecido.

O estudo foi conduzido na cidade de Viçosa, MG. A coleta de dados foi realizada com uma amostra intencional aleatória, composta de 292 consumidores abordados em dois supermercados da região central da cidade. Foram aplicados questionários estruturados com abordagem quantitativa na qual se utilizou a técnica survey. A fim de evitar a tendenciosidade dos resultados, os entrevistados responderam as questões por meio de cartões circulares contendo as respostas (GIL, 2002; MALHOTRA, 2006). O tamanho da amostra foi definido pela Equação 1 (TRIOLA, 2005).

$$
\mathrm{n}=\frac{\mathrm{qp} Z^{2}}{\mathrm{E}^{2}}
$$

em que: $n$ = tamanho da amostra; $Z=1,96$ (abscissa da normal a um nível de confiança de 95\%); $p=0,5$ (variabilidade máxima estimada); $q=1-p$; $\mathrm{E}=0,10$ (nível de precisão).

A escolha da cidade e dos supermercados onde foram aplicados os questionários baseou-se na grande população universitária, que prima pela praticidade nos hábitos alimentares e que habita o município, visto que a cidade gravita em torno de uma universidade federal.

Os dados foram analisados pelo software SPSS. Realizou-se a correlação de Spearmam para verificar as correlações entre o consumo de sucos prontos e as variáveis gênero, renda, escolaridade, idade e estado civil, assim como o interesse pelo produto e as variáveis opinião sobre o produto e motivação para o consumo. As variáveis utilizadas neste estudo foram: marca e sabor (variáveis independentes) e preferência do consumidor (variável dependente).

\section{Resultados e discussão}

\subsection{Características demográficas dos consumidores}

A faixa etária predominante da população entrevistada foi de 20 a 30 anos (40\%, n = 117), sendo $58 \%(n=171)$ do sexo masculino, $64 \%$ solteiros $(n=187)$ e $51 \%$ estudantes $(n=149)$. Em relação ao grau de instrução, $51 \%(n=149)$ tinham curso superior incompleto e 49\% ( $n=143)$ declararam ter renda familiar entre 10 a 21 salários-mínimos (Figura 1).

\subsection{Hábitos de consumo}

Na Figura 2 estão apresentados os dados sobre os hábitos de consumo de suco pelos entrevistados. Do total dos entrevistados, $32 \%(n=92)$ consomem algum tipo de suco de três a cinco vezes por semana, principalmente junto ao almoço e/ou jantar $(42 \%, n=123)$.

A maioria dos participantes $(72 \%, n=211)$ prefere o suco tradicional ao light/diet (Figura 2).

Não foi observada preferência em relação ao tipo de suco, se industrializado ou natural, visto que $53 \%(n=156)$ consomem ambos (Figura 2). Quanto ao sabor, a primeira opção mais citada foi o de laranja, seguido pelos de uva, goiaba, maracujá e manga (Figura 3). O suco de laranja, comumente encontrado sob forma pasteurizada, para preservar o sabor (SOUZA, 2013), é o suco de fruta mais consumido mundialmente. 
Caracterização do mercado consumidor de sucos prontos para o consumo

CARMO, M. C. L. et al.

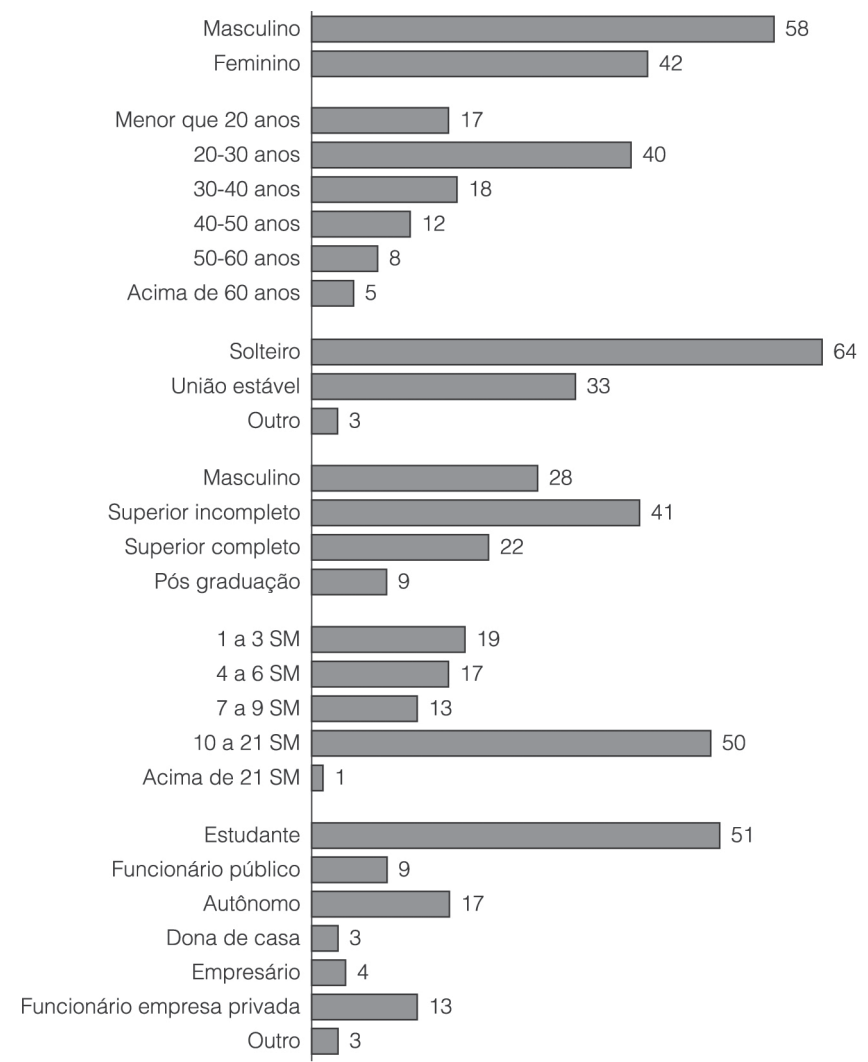

Figura 1. Frequência das características demográficas dos consumidores de suco $(n=292)$ da cidade de Viçosa, expressa em porcentagem. $\mathrm{SM}=$ salário mínimo.
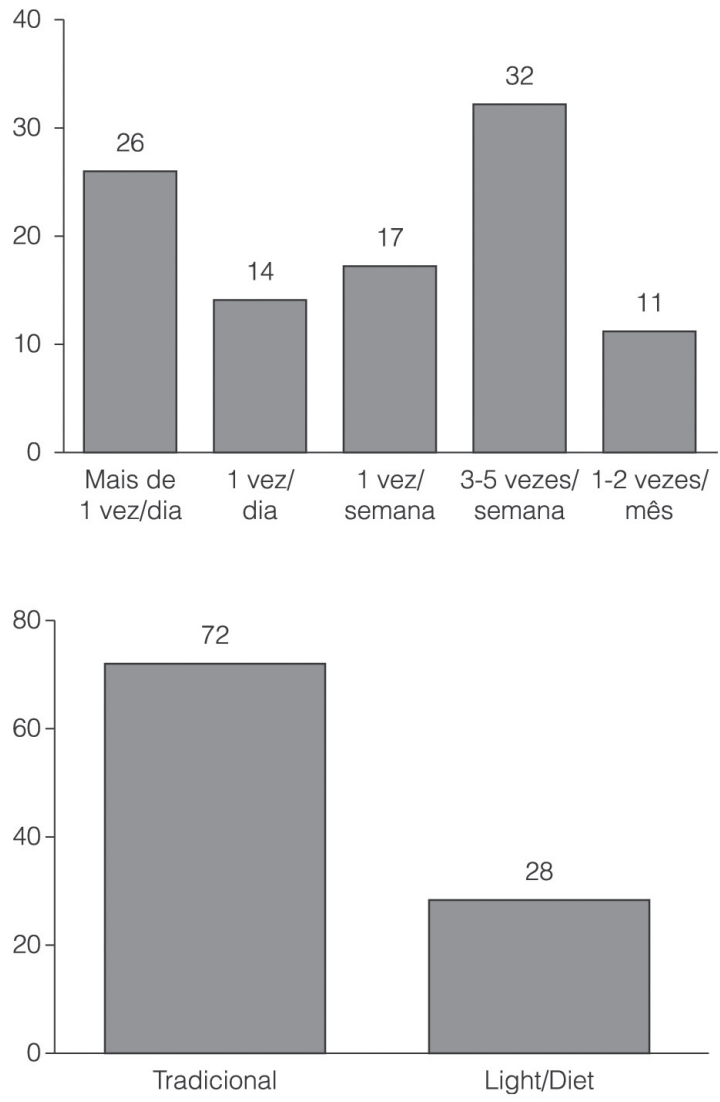

\subsection{Comportamento dos consumidores de sucos industrializados}

Sabor, qualidade e validade foram critérios considerados extremamente importantes na escolha de sucos industrializados pela maioria dos entrevistados. Cerca de $45 \%(n=133)$ incluíram o preço nessa categoria, enquanto a embalagem e a praticidade foram consideradas muito importantes por $39 \%(n=115)$ e $44 \%(n=130)$ dos entrevistados, respectivamente. Apenas $33 \%(n=98)$ dos participantes consideraram o valor nutricional e a marca como muito importantes na aquisição de sucos. Percebe-se que a praticidade também é um fator considerado fonte de satisfação entre os pesquisados, uma vez que $70 \%$ deles responderam ser esse era um fator muito importante ou extremamente importante (Tabela 1).

Metade dos consumidores afirma ler as informações nutricionais contidas nos rótulos.

\subsection{Motivação para o consumo}

As marcas preferidas foram a Del Valle $(22 \%$; $n=63)$ e Tial (20\%; $n=59)$ (Figura 4).

Sabor, qualidade e confiança na marca foram os principais quesitos apontados para a preferência por ela. Esse resultado é semelhante ao descrito no estudo de Sartório (2006), no qual os fatores para a decisão do consumidor foram qualidade, preço e marca (Figura 5).
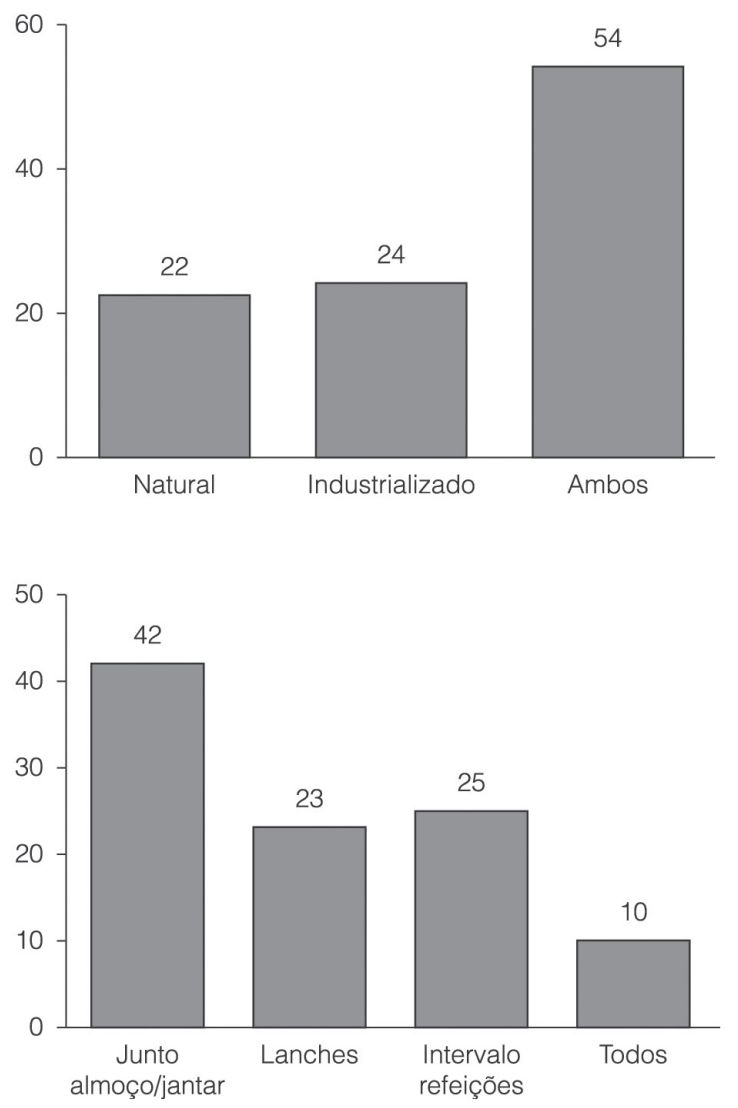

Figura 2. Perfil do hábito de consumo de sucos, expresso em porcentagem. 
Caracterização do mercado consumidor de sucos prontos para o consumo

CARMO, M. C. L. et al.

Tabela 1. Critérios a serem considerados no grau de interesse dos consumidores.

\begin{tabular}{|c|c|c|c|c|c|c|c|c|}
\hline $\begin{array}{c}\text { Grau de } \\
\text { importância }\end{array}$ & $\begin{array}{c}\text { Embalagem } \\
(\%)\end{array}$ & $\begin{array}{c}\text { Praticidade } \\
(\%)\end{array}$ & $\begin{array}{c}\text { Validade } \\
\text { (\%) }\end{array}$ & $\begin{array}{c}\text { Preço } \\
(\%)\end{array}$ & $\begin{array}{c}\text { Qualidade } \\
(\%)\end{array}$ & $\begin{array}{c}\text { Sabor } \\
(\%)\end{array}$ & $\begin{array}{c}\text { Valor } \\
\text { nutricional } \\
(\%)\end{array}$ & $\begin{array}{c}\text { Marca } \\
(\%)\end{array}$ \\
\hline $\begin{array}{l}\text { Totalmente sem } \\
\text { importância }\end{array}$ & 5 & 4 & 2 & 4 & 0 & 1 & 10 & 10 \\
\hline Pouco importante & 11 & 8 & 3 & 1 & 0 & 2 & 12 & 13 \\
\hline $\begin{array}{l}\text { De alguma } \\
\text { importância }\end{array}$ & 22 & 18 & 7 & 15 & 8 & 5 & 19 & 21 \\
\hline Muito importante & 39 & 44 & 29 & 35 & 29 & 26 & 33 & 33 \\
\hline $\begin{array}{l}\text { Extremamente } \\
\text { importante }\end{array}$ & 23 & 26 & 59 & 45 & 63 & 66 & 26 & 23 \\
\hline
\end{tabular}

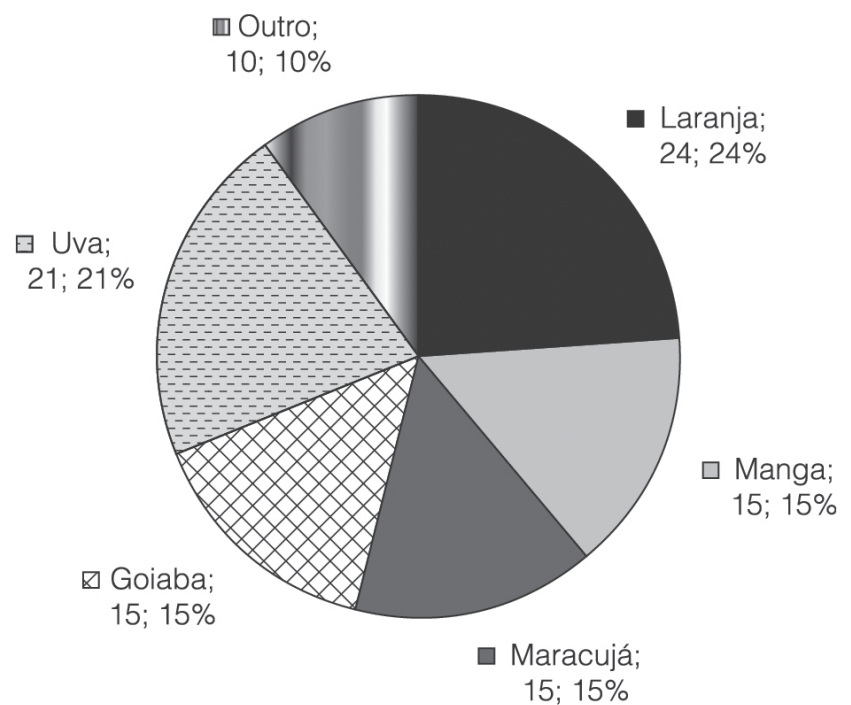

Figura 3. Preferência dos consumidores de Viçosa $(n=292)$ em relação ao sabor do suco, expressa em porcentagem.

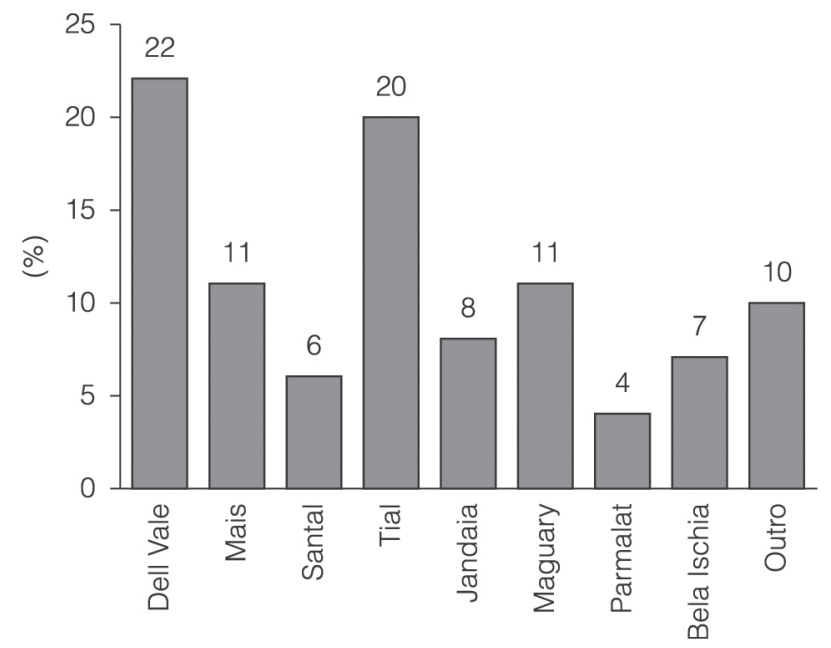

Figura 4. Marcas de sucos industrializados mais consumidas entre os entrevistados.

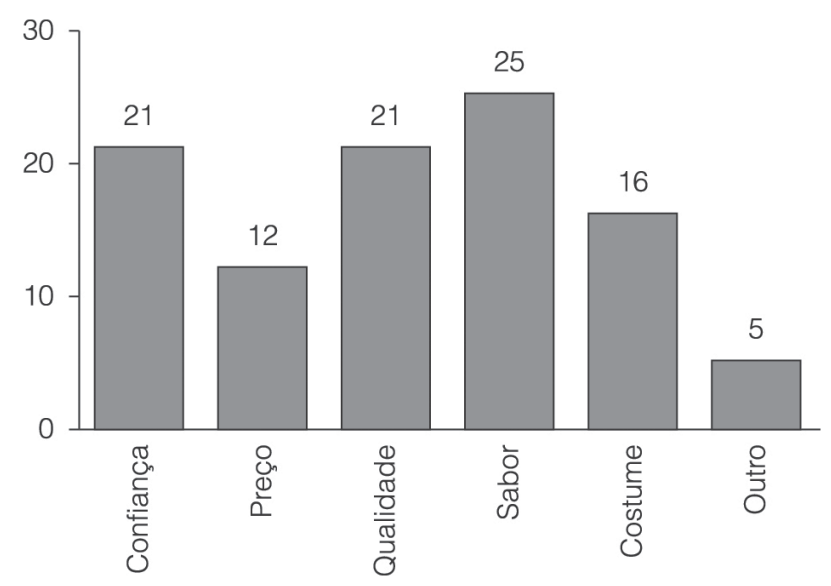

Figura 5. Motivação para o consumo, expressa em porcentagem.

\section{Conclusão}

Ficou demonstrado que a maior parte dos entrevistados consome sucos de três a cinco vezes por semana em suas refeições. Na hora da escolha, o sabor, a qualidade e a validade são mais importantes que a praticidade. Não foi observada preferência em relação ao tipo de suco, se industrializado ou tradicional. Se light ou diet, isso não é uma preocupação, ainda, entre os consumidores, a maioria prefere os naturais. Embora o mais consumido seja o suco de laranja, a procura por sabores diversificados, principalmente uva, goiaba, maracujá e manga, é grande.

Portanto, o mercado de sucos apresenta um enorme potencial a ser explorado principalmente entre os jovens que, cada vez mais exigentes, começam a mudar seus hábitos em busca de uma vida mais saudável. 
Caracterização do mercado consumidor de sucos prontos para o consumo

CARMO, M. C. L. et al.

\section{Referências}

DAGEVOS, H. Consumers as four-faced creatures. Looking at food consumption from the perspective of contemporary consumers. Appetite, v. 45, n. 1, p. 32-39, 2005.

DIAS, A. D. M. et al. A importância do planejamento estratégico de marketing aplicado nas pequenas empresas. In: SEMINÁRIO DE PRODUÇÃO ACADÊMICA DA ANHANGUERA, 3., 2013. Anais... [S. I.]: Anhanguera Educacional, p. 101-106, 2013.

ENDO, E.; BERTOLDI, M. C.; PINHEIRO, N. M. S.; ARRUDA, A. C.; MININ, V. P. R. Caracterização do mercado consumidor de" água aromatizada": hábitos e motivações para o consumo. Ciência e Tecnologia de Alimentos, v. 29, n. 2, p. 365-70, 2009. http://dx.doi.org/10.1590/S0101-20612009000200020

FERRAREZI, A. C. Interpretação do consumidor, avaliação da intenção de compra e das características físico-químicas do néctar e do suco de laranja pronto para beber. 2008. $104 \mathrm{f}$. (Mestrado em Ciência dos Alimentos)-Faculdade de Ciências Farmacêuticas, Universidade Estadual Paulista, 2008.

FERREIRA, K. A.; ALCÂNTARA, R. L. C. Approaches for implementation of the postponement strategy: a multicase study in the food industry. Gestão \& Produção, v. 20, n. 2, p. 357-372, 2013.

GIL, A. C. Como elaborar projetos de pesquisa. 4. ed. São Paulo: Atlas, 2002.

MACHADO, J. G. D. C. F. Estratégias de marketing na indústria de amendoim: um estudo em empresas da Alta Paulista. Latin
American Journal of Business Management, v. 3, n. 2, p. 21-29, 2013.

MALHOTRA, N. K. Pesquisa de marketing: uma orientação. [S. I.]: Bookman, 2006.

SARTÓRIO, A. Os fatores que influenciam a compra dos sucos prontos industrializados. 2006. 70 f. (Trabalho de Conclusão de Curso)-Centro Universitário de Brasília, Brasília, 2006. PMCid:PMC2546487

SERVIÇO BRASILEIRO DE APOIO ÀS MICRO E PEQUENAS EMPRESAS - SEBRAE. Mercado de sucos prontos para beber cresce 7,2\%. 2006. Disponível em: <http://www.sebrae-sc. com.br/newart/default.asp?materia $=6727=>$. Acesso em: 15 maio 2013.

SOLOMON, M. R. Comportamento do consumidor. Porto Alegre: Artmed, 2008.

SOUZA, C. D. Estudo bibliométrico da produção científica do setor citrícola no Brasil. Liinc em Revista, v. 9, n. 1, p. 55-60, 2013.

TRIOLA, M. F. Introdução à estatística. Rio de Janeiro: LTC, 2005.

WANSINK, B. Environmental factors that increase the food intake and consumption volume of unknowing consumers. Annual Reviews of Nutrition, v. 24, p. 455-479, 2004. PMid:15189128. http://dx.doi.org/10.1146/annurev.nutr.24.012003.132140 